\title{
Semiclassical environment of collapsing shells
}

\author{
Kinjal Banerjet* \\ IUCAA, Post Bag 4, Ganeshkhind, Pune - 411 00\%, India \\ Aseem Paranjape栶 \\ Tata Institute of Fundamental Research, Homi Bhabha Road, \\ Colaba, Mumbai - 400 005, India
}

(Dated: November 5, 2018)

\begin{abstract}
We explore in detail the semiclassical environment of collapsing shells of matter, and determine the semiclassical flux measured by a variety of observers. This study is a preliminary step in a broader investigation of thermodynamic properties of the geometry of collapsing objects. Specifically, in this paper we consider spherically symmetric null and timelike collapsing shells which form an event horizon, and calculate the flux measured by observers both inside and outside the shell, and both inside and outside the event horizon, and find nontrivial results in most of the cases. Additionally, we also investigate the environment of a shell which collapses but does not form a horizon, halting at some radius larger than the Schwarzschild radius, and find that such an object generically gives rise to a pulse of radiation which is sharply peaked as it travels inwards and is reflected at the origin, and eventually emerges from the shell in a "thermalized" form. Our results have potential consequences in addressing questions pertaining, e.g. to black hole entropy and backreaction.
\end{abstract}

\section{INTRODUCTION}

The behaviour of quantum fields in the spacetime geometry of black holes and gravitationally collapsing objects in general has been a subject of detailed investigation since Hawking's seminal discovery [1] of a nearly thermal flux of particles that is measured by observers located outside the collapsing object. Several authors have subsequently contributed towards building an understanding of the semiclassical environment of collapsing objects [2, 3, 4, [5]. One of the most fascinating aspects of this field of research is the fact that classical black holes appear to behave like macroscopic thermodynamical objects [6]. An important offshoot of this behaviour is the so called information paradox (see, e.g. 7] for a recent interesting discussion), and an understanding of this thermodynamics at the quantum level is also likely to have consequences towards building a quantum theory of gravity [8].

There are several questions that can be asked within the semiclassical framework. For example, considering a Schwarzschild black hole for simplicity, it is a celebrated fact that the black hole can be attributed an entropy which scales as one quarter of the area of the event horizon in Planck units. This entropy however has a physical relevance only to those observers who choose to remain outside $r=2 M$ forever, i.e. - for observers who truly experience $r=2 M$ as an event horizon. Since forever is a long time to wait, it becomes interesting to ask whether the concepts of black hole entropy etc. can be meaningfully addressed locally. For example, what entropy (if any) would be attributed to the surface $r=2 M$ by observers who remain outside for an arbitrarily long time, before suddenly choosing to jump into the black hole? Such observers will clearly eventually have access to many more microstates compared to their cousins who choose to remain outside forever, but until the decision to fall in is actually made, there are no grounds to distinguish between these two classes.

Returning to the context of collapsing objects, further complications arise when one begins to account for the backreaction of the semiclassical flux of particles, on the classical geometry of the collapsing object. The basic idea here is that the loss of energy from the collapsing object (which we take to be spherically symmetric, for simplicity), in the form of the semiclassical flux, implies that the correct background geometry in the exterior of the object must be time dependent, rather than the static Schwarzschild spacetime [4, 5, 9, 10]. Due to technical challenges, the exact effect of the backreaction has been a subject of much debate, with claims to the extent that the formation of the event horizon itself may be hindered by the backreaction [11, 12]. While this appears unlikely [13], it is nevertheless

$\dagger$ Address from 8th Oct. 2009: The Abdus Salam ICTP, Strada Costiera 11, 34014 Trieste, Italy.

*Electronic address: kinjal@iucaa.ernet.in

‡Electronic address: aparanja@ictp.it 
true that determining the exact geometry in the late stages of black hole evaporation, remains an open problem. Consequently, questions regarding the thermodynamic behaviour of the geometry of the black hole/collapsing object also remain clouded by the backreaction.

In this paper, as a preliminary step towards addressing the broader issues raised above, we set a simpler mandate for ourselves. We wish to explore the full semiclassical environment of a collapsing object, which we will consider to be a spherically symmetric thin shell [2]. Classically, the exterior of the shell is described by Schwarzschild spacetime while the interior is Minkowski spacetime. In particular we wish to determine the semiclassical flux, as encoded in the renormalized stress tensor, observed by a variety of observers, both inside and outside the shell, before and after horizon formation.

There are several motivating factors behind such a study : Firstly, while the flux seen by observers outside the shell and the event horizon has been studied in great detail (beginning with Hawking's own calculation), the nature of the flux inside the shell, and inside the event horizon but outside the shell, remains largely unexplored. In fact, an argument one usually encounters is that observers who fall into a black hole do not encounter a significant flux. Our calculation assumes zero backreaction, and we will see that in this case an infalling observer will see a flux of outgoing particles which in fact increases without bound as the observer approaches the singularity (although as expected it is finite at all other locations, including $r=2 M$ ). Further, at least in the case of timelike collapse, we find that observers inside the shell also see a flux of particles, which might have some interesting consequences which we will return to discuss in the final section.

Apart from collapsing shells which form an event horizon, we will also discuss the case, first described in Ref. [13], of a shell which even classically does not form a horizon; instead it asymptotically approaches a final radius larger than $2 M$. In Ref. [13] such a shell was shown to have very interesting properties, although that discussion was limited partly to order of magnitude estimates, since some required expressions were not analytically obtainable. In this paper we will perform a full numerical study of such a shell trajectory, and also explore its semiclassical behaviour both inside and outside the shell. We will find that such trajectories are generically expected to generate a pulse of radiation which travels inwards, reflects at the center and travels back outwards, eventually reaching an outside observer in a "thermalized" form (to be made precise later).

At this stage, it is worth addressing why we choose to study the seemingly unrealistic situation of a collapsing shell. The first reason of course is that the geometry of a shell is simple to handle analytically, not least because the interior is just Minkowski spacetime. A second important reason is that the absence of interior matter allows us to study the purely gravitational aspects of quantum fields in the interior. It is important to understand such effects before addressing the problem of quantum fields whose semiclassical effects interact with those of classical matter inside a star for example. Throughout our discussion we will simply prescribe the classical trajectory of the shell, and not worry about the specific (singular) stress-tensor needed to generate the trajectory. This is not expected to interfere with the conclusions we draw regarding the semiclassical behaviour of the shell.

The plan of the paper is as follows : In Section 1 we start with the example of a null shell which has been collapsing forever. While such a geometry has been studied in the literature [4, 14, 15], we note that the region of Schwarzschild spacetime for $r<2 M$ has been largely ignored. Another reason to study the null shell is that all expressions for the flux, for all observers, can be obtained analytically, with no numerical inversions involved. This section will therefore serve as a useful warmup to the more involved calculations for timelike trajectories. In Section [II] we turn to studying timelike trajectories, where many of the calculations will be numerical. We will first study a trajectory which forms a horizon, describing the flux seen by various observers, and then turn to the trajectory mentioned earlier, which asymptotically halts without forming a horizon. We will end in Section IV with a brief discussion and prospects for future work.

\section{A. Notation}

To avoid confusion, in this subsection we will lay down all the notation that will be used subsequently and in the next subsection we recapitulate the prescription for calculating the semiclassical flux in a given geometry. Note that all our calculations assume zero backreaction.

We will be assuming Planck units throughout, with $G, \hbar$ and $c$ set to unity. We will mostly follow the notation used in Ref. [13], which in turn was based on the notation in the review by Brout et al. [9]. Additionally, to avoid cumbersome factors of $2 M$ (where $M$ is the mass of the collapsing object), we will assume that all the coordinates are non-dimensionalized by dividing out by $2 M$. This will lead to expressions for the flux, etc. with factors of $2 M$ missing, which can be put back appropriately by dimensional arguments. Latin tensor indices $a, b, .$. range over $0 . .3$, and we use the mostly plus sign convention.

As mentioned earlier, the exterior of the shell is Schwarzschild spacetime while the interior is Minkowski, and we can use the same radial coordinate $r$ to foliate both regions. We refer to Schwarzschild time as $t$ and Minkowski time 
as $T$. Outgoing null coordinates will be a variation of the letter $u$, similarly ingoing coordinates and $v$. To be precise, the interior Minkowski null coordinates will be

$$
V=T+r ; \quad U=T-r,
$$

and the exterior Eddington null coordinates will be

$$
v=t+x_{*}(r) ; u=t-x_{*}(r)
$$

where we have defined the tortoise function

$$
x_{*}(r)=r+\ln |r-1|,
$$

(which should be read as $x_{*}(r) / 2 M=r / 2 M+\ln |r / 2 M-1|$ ). All "Kruskalized" (i.e. - exponentiated) versions of the null coordinates will be referred to using calligraphic fonts. Specifically, $\mathcal{U}$ and $\mathcal{V}$ will denote the standard Kruskal null coordinates

$$
\mathcal{V}=2 e^{v / 2} ; \mathcal{U}=\mp 2 e^{-u / 2} ; \mathcal{U} \mathcal{V}=4 e^{r}(1-r)
$$

with the upper $\operatorname{sign}$ in $\mathcal{U}$ for $r>1$ (the Schwarzschild radius being unity in non-dimensionalized form) and the lower sign for $r<1$. Other Kruskalized coordinates will be prescribed as needed.

With this setup, the (non-dimensionalized) metric in various regions and in various coordinates becomes (suppressing the line element for the 2-sphere, which is always $\left.r^{2} d \Omega^{2}\right)$,

$$
\begin{aligned}
d s_{\mathrm{int}}^{2} & =-d T^{2}+d r^{2}=-d U d V \\
d s_{\mathrm{ext}}^{2} & =-(1-1 / r) d t^{2}+(1-1 / r)^{-1} d r^{2} \\
& =-\left(1-\frac{1}{r}\right) d u d v=-\frac{e^{-r}}{r} d \mathcal{U} d \mathcal{V} .
\end{aligned}
$$

The subscript $s$ will always refer to evaluation on the shell, so the trajectory is $r=R_{s}$, $v=v_{s}$, etc. The specific parameter used along the trajectory will be chosen according to convenience, and could be one of the coordinates.

\section{B. Recap of semiclassical prescription}

Throughout, we will be interested in the semiclassical flux $\mathcal{F}=n_{a}\left\langle T_{b}^{a}\right\rangle u^{b}$ measured by an observer with 4-velocity $u^{a}$ where $n_{a} u^{a}=0, n_{a} n^{a}=1=-u_{a} u^{a}$, and $\left\langle T_{a b}\right\rangle$ is the renormalized stress-tensor vacuum expectation value (VEV) of a quantized, massless scalar field in a specific vacuum state. We refer the reader to Refs. [3, 9] for detailed treatments of the formalism, and to Ref. [13] for a pedagogical introduction. The Hawking flux has the value $(\pi / 12) T_{H}^{2}=(192 \pi)^{-1}(2 M)^{-2}$, where $T_{H}=1 / 8 \pi M$ is the Hawking temperature. The vacuum state $\mid$ in $\rangle$ to be used, is completely defined by specifying its behaviour at past null infinity $\mathcal{I}^{-}$and by requiring that its mode functions vanish at reflection at the origin $r=0$. Typically one requires that the vacuum on $\mathcal{I}^{-}$be identical to the Minkowski vacuum, so that the ingoing modes of the state $\mid$ in $\rangle$ become $\sim e^{-i \omega v}$ for a typical timelike collapse. We will see that the situation for a null shell collapsing forever, is somewhat different, since in this case the portion of $\mathcal{I}^{-}$relevant for thermal flux in the exterior, lies inside the shell. However, here also we will be able to meaningfully compute the flux observed by specific classes of observers.

An important assumption we make is to work in the $1+1 s$-wave approximation, ignoring the Schwarzschild potential barrier, so that the results of 2-dimensional conformal field theory (CFT) apply. For a discussion of this approximation and its validity, see the review by Brout et al. [9]. In this approximation, for a vacuum state |vac with ingoing and outgoing modes given respectively by $e^{-i \omega f_{-}}$and $e^{-i \omega f_{+}}$, such that the metric in the relevant region in terms of these null functions is $d s^{2}=-C\left(f_{+}, f_{-}\right) d f_{-} d f_{+}$, the components of the renormalized stress-tensor VEV in the state $|\mathrm{vac}\rangle$ are calculated as

$$
\left\langle T_{f_{-} f_{-}}\right\rangle_{\mathrm{vac}}=-F_{f_{-}}\left[C\left(f_{-}, f_{+}\right)\right] ;\left\langle T_{f_{+} f_{+}}\right\rangle_{\mathrm{vac}}=-F_{f_{+}}\left[C\left(f_{-}, f_{+}\right)\right] \text {, }
$$

where the derivative operator $F$ is defined, for any function $f(x)$, as

$$
F_{x}[f(x)] \equiv \frac{1}{12 \pi} f^{1 / 2} \partial_{x}^{2} f^{-1 / 2}=-\frac{1}{48 \pi}\left[2 \partial_{x}^{2} \ln f-\left(\partial_{x} \ln f\right)^{2}\right] .
$$




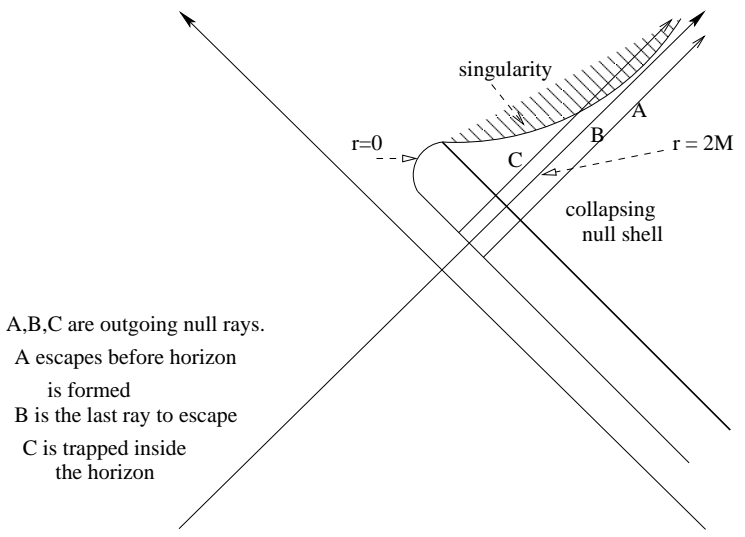

FIG. 1: Penrose diagram for the null shell that has been collapsing for all time [14]. Ingoing rays have $\mathcal{V}=$ const and outgoing rays have $\mathcal{U}=$ const.

The cross terms $\left\langle T_{f_{-} f_{+}}\right\rangle_{\mathrm{vac}}$ can be determined by requiring that the stress tensor be covariantly conserved. The following relations are useful while analyzing the flux in different regions :

$$
\begin{aligned}
F_{x}[x] & =\frac{1}{16 \pi x^{2}}, \\
F_{x}[1 / f(x)] & =-F_{x}[f(x)]+\frac{1}{24 \pi}\left(\partial_{x} \ln f\right)^{2}, \\
F_{x}[f(x) g(x)] & =F_{x}[f(x)]+F_{x}[g(x)]+\frac{1}{24 \pi}\left(\partial_{x} \ln f\right)\left(\partial_{x} \ln g\right), \\
F_{s}[f(x(s))] & =\frac{1}{s^{\prime 2}}\left(F_{x}[f(x)]+\frac{1}{24 \pi}\left(\partial_{x} \ln f\right)\left(\partial_{x} \ln s^{\prime}\right)\right),
\end{aligned}
$$

where in the last equation, $s^{\prime}=d s / d x$.

Hawking's result follows from recognizing that for a generic timelike collapse, requiring the vacuum $\mid$ in $\rangle$ to be Minkowskian at $\mathcal{I}^{-}$, the corresponding modes in the exterior region become $e^{-i \omega v}, e^{-i \omega G(u)}$, where $G(u)$ is that value $v=G$ for which an ingoing ray of constant $G$, becomes a ray of constant $u$ after being reflected at the origin and emerging from the shell. Further, one finds that due to the presence of the event horizon, the generic behaviour of $G(u)$ for $u \rightarrow \infty$ is $d G / d u \sim e^{-u / 2}$, which leads to the result that in the state $\mid$ in $\rangle$, an exterior observer using the Eddington $u, v$ coordinates measures a constant outgoing thermal flux $\left\langle T_{u u}\right\rangle-\left\langle T_{v v}\right\rangle=(\pi / 12) T_{H}^{2}$ as $u \rightarrow \infty$. [The notation $\left\langle T_{a b}\right\rangle$ with no subscript will always refer to the vacuum state $\mid$ in $\rangle$.] We will see this result in the trajectories we study. For further details see Refs. [3, [9, 13].

\section{NULL SHELL}

We begin by considering a null shell that has been collapsing for all time [4, 14, 15]. The global structure of this system is different from the usual timelike collapse situations which we will discuss in Section III, since a part of $\mathcal{I}^{-}$in this case lies inside the shell. Since the matching of metrics in (5) occurs along a line of constant $V$ (which we conveniently choose to be $V=0$ ), there is a freedom in relating the exterior and interior ingoing coordinates. We exploit this freedom to set $\mathcal{V}_{\mathrm{m}} \equiv 2 e^{V / 2}=\mathcal{V}$. Additionally, we use the Kruskalized outgoing coordinate $\mathcal{U}_{\mathrm{m}} \equiv 2 e^{-U / 2}(1+U / 2)$, and it is then easy to show that on the shell (i.e. - at $\mathcal{V}_{\mathrm{m}}=\mathcal{V}=2$ ) we have $\mathcal{U}_{\mathrm{m}}=\mathcal{U}$ for all $r$. In other words, we can use the usual Kruskal coordinates $\mathcal{U}, \mathcal{V}$ over the entire spacetime, with the metric given by

$$
\begin{aligned}
& d s_{\mathrm{int}}^{2}=-d U d V=-\frac{2 e^{-r}}{(-U)} d \mathcal{U} d \mathcal{V}, \\
& d s_{\mathrm{ext}}^{2}=-\frac{e^{-r}}{r} d \mathcal{U} d \mathcal{V},
\end{aligned}
$$

with the event horizon $r=1$ corresponding to the outgoing ray $\mathcal{U}=0$ or $U=-2$. Figure 1 shows the Penrose diagram for this spacetime [14]. 


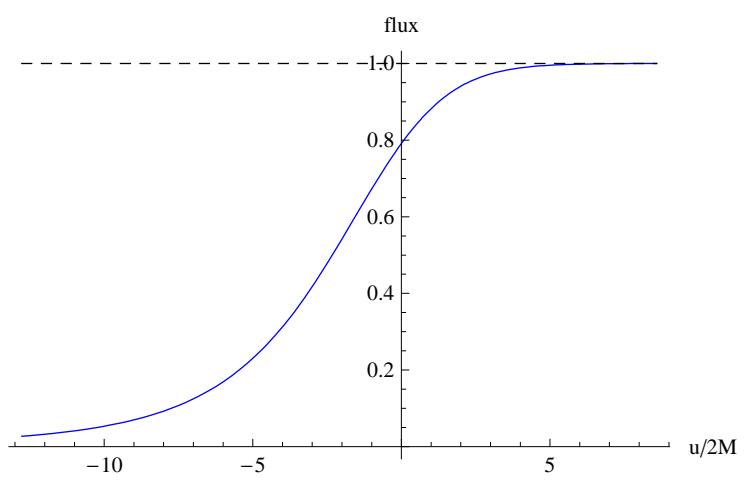

FIG. 2: The semiclassical flux (normalized by the Hawking value) measured by static observers outside the event horizon, for the null shell trajectory.

In the usual timelike collapse situations where the shell would typically start collapsing from some finite radius, $\mathcal{I}^{-}$ lies entirely outside the shell and the spacetime is asymptotically flat. It is then natural to choose a vacuum whose ingoing modes at $\mathcal{I}^{-}$are $\sim e^{-i \omega v}, v$ being the ingoing Eddington coordinate. In the present situation, it is natural to define the vacuum in the interior region of $\mathcal{I}^{-}$using the Minkowski ingoing coordinate $V$. Note however that our choice of mapping the ingoing coordinates between the interior and exterior, ensures that $V=v$ at the boundary, and hence the $\mid$ in $\rangle$ vacuum can still be chosen to have modes $\sim e^{-i \omega v}=e^{-i \omega V}$ on all of $\mathcal{I}^{-}$. The reflection condition at $r=0$ then fixes the outgoing modes of $\mid$ in $\rangle$ to be $\sim e^{-i \omega U}$ in the interior region. Equivalently, the function $G$ discussed in Section $\amalg \mathrm{IB}$ is simply $G(U)=U$ in the interior. It then follows that the renormalized stress tensor in the state $\mid$ in $\rangle$ is identically zero in the interior of the shell, since the conformal factor in the interior is constant in the $U, V$ coordinates.

In the exterior, for $r>1$ we want the flux measured by static observers $\mathcal{O}_{1}$ who use the Eddington $u, v$ coordinates. The function $G(u)$ is now determined by the implicit equation

$$
-e^{-u / 2}=e^{-G / 2}(1+G / 2) .
$$

The stress tensor components in the state $\mid$ in $\rangle$ are given by

$$
\left\langle T_{v v}\right\rangle=-\left(\frac{\pi}{12} T_{H}^{2}\right) \frac{1}{r^{3}}\left(4-\frac{3}{r}\right) ;\left\langle T_{u u}\right\rangle=\left\langle T_{v v}\right\rangle+F_{u}\left[\frac{d G}{d u}\right] .
$$

[Note that the $r$-dependent part of these expressions is simply the static contribution in the so called Boulware state.] The outgoing flux measured by observers $\mathcal{O}_{1}$ is

$$
\begin{aligned}
\mathcal{F}=-\left\langle T_{t}^{r}\right\rangle & =\left\langle T_{u u}\right\rangle-\left\langle T_{v v}\right\rangle \\
& =F_{u}\left[\frac{d G}{d u}\right]=-\frac{1}{12 \pi} \frac{1}{G(u)^{4}}(3+2 G(u)) .
\end{aligned}
$$

We can analytically see that as $u \rightarrow \infty, G \rightarrow-2, d G / d u \sim e^{-u / 2}$, and hence the flux asymptotically approaches

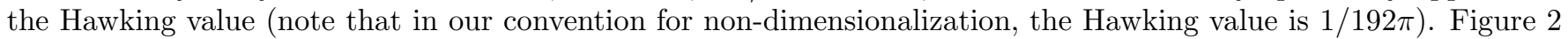
shows this flux (normalized by the Hawking value) as a function of $u$.

An interesting region to consider is the exterior of the shell for $r<1$. In this region it is more natural to use the Kruskal coordinates $\mathcal{U}, \mathcal{V}$, since this region is likely to be accessed by freely falling observers $\mathcal{O}_{2}$. The stress tensor (in the entire exterior region $r>R_{s}$ ) in these coordinates is

$$
\begin{aligned}
& \left\langle T_{\mathcal{V V}}\right\rangle=\frac{4}{\mathcal{V}^{2}}\left\langle T_{v v}\right\rangle, \\
& \left\langle T_{\mathcal{U U}}\right\rangle=\frac{4}{\mathcal{U}^{2}}\left\langle T_{u u}\right\rangle,
\end{aligned}
$$

Although superficially divergent, the expression for $\left\langle T_{\mathcal{U} \mathcal{U}}\right\rangle$ is actually finite on the horizon $\mathcal{U}=0$. The structure of $\left\langle T_{v v}\right\rangle$ and $\left\langle T_{u u}\right\rangle$ shows, on the other hand, that observers who fall into the singularity will generically see a flux that increases without bound. We will see this feature again when studying timelike trajectories in Section 
where we will display a more detailed expression and a plot for the flux seen by observers $\mathcal{O}_{2}$. A third class $\mathcal{O}_{3}$ which remains in free fall inside the shell for a certain time, does not see a flux in the present null case. The timelike case however will prove to be different.

We end this section by noting that although inertial observers in the interior of the null shell do not see a semiclassical flux, there is a natural class of accelerated observers who do see a flux. These are observers following trajectories picked out by the Kruskal null coordinates, and are related to Rindler observers in the Minkowski region. (Notice the contrast with the exterior, where such Kruskal observers are inertial, while observers following trajectories picked out by Eddington coordinates are Rindler-like.) For a vacuum state $\mid$ vac $\rangle$ defined by the modes $e^{-i \omega \mathcal{V}}, e^{-i \omega \mathcal{U}}$ in the interior, the stress-tensor components are given by

$$
\begin{aligned}
\left\langle T_{\mathcal{V} \mathcal{V}}\right\rangle_{\mathrm{vac}} & =\left(\frac{\pi}{12} T_{H}^{2}\right) e^{-V}, \\
\left\langle T_{\mathcal{U} \mathcal{U}}\right\rangle_{\mathrm{vac}} & =\left(\frac{\pi}{12} T_{H}^{2}\right) \frac{4 e^{U}}{U^{4}}\left(U^{2}-4 U+12\right) .
\end{aligned}
$$

\section{TIMELIKE SHELL}

We now turn to the more realistic situation of a shell collapsing along a timelike trajectory. We will discuss two types of trajectories : the first is a "conventional" trajectory in which the shell forms a horizon in finite proper time, and continues to collapse to a singularity. The second is an "asymptotic" trajectory, in which the shell asymptotically approaches a final radius larger than its Schwarzschild radius, so that a horizon is never formed. In Ref. [13] the latter was shown to have very interesting behaviour, in that an outside observer would see a constant Hawking-like flux for some finite time interval, after which the flux would exponentially decay to zero. In Ref. [13] however, only a part of the flux was explicitly calculated, with order of magnitude estimates for the remaining part, since the functional inversions needed could not be performed analytically. Below we will show the results of a full numerical calculation of the flux, which will corroborate the results of Ref. [13]. We will also see some additional effects as mentioned in the introduction, which are only revealed in the numerical analysis. All numerical calculations were performed on Mathematica. We begin with a discussion of the conventional horizon-forming trajectory.

\section{A. Horizon-forming trajectory}

We parametrize the trajectory using the shell proper time. Our trajectory is $r=R_{s}(\tau)$ (assuming a nondimensionalised form for all coordinates), where

$$
R_{s}^{\prime 2}=\theta(\tau) \frac{f(\tau)}{R_{s}} ; R_{s}(\tau)=R_{0}, \quad \tau \leq 0,
$$

where a prime denotes a derivative with respect to the argument $\tau, \theta$ is the Heaviside step function, and $f$ is a function which is chosen so that $f \rightarrow 1$ at late times. Note that $R_{s}^{\prime} \leq 0$. The trajectory therefore remains at a constant radius $R_{0}$ until $\tau=0$, and eventually becomes a pseudo-free-fall curve, mimicking a particle freely falling in the potential of a body of mass $M$. The specific form of $f$ we choose is

$$
f(\tau)=1-e^{-(\kappa \tau)^{a}},
$$

with $\kappa$ and $a$ as parameters. We will fix $a=5$, which ensures that $R_{s}(\tau)$ has a continuous third derivative at $\tau=0$ (and hence for all $\tau<\tau_{\max }$, where $R_{s}\left(\tau_{\max }\right)=0$ ). Since the parametrization is in terms of the shell's proper time, the equations (5) are sufficient to completely determine the trajectory in terms of other coordinates $u$, $v$, etc. as well. In particular, the functions $v_{s}, U_{s}$ are useful in the analysis, and can be obtained by integrating the following expressions

$$
\begin{aligned}
v_{s}^{\prime} & =1 / g ; g(\tau) \equiv-R_{s}^{\prime}+\sqrt{R_{s}^{\prime 2}+1-1 / R_{s}}, \\
U_{s}^{\prime} & =-R_{s}^{\prime}+\sqrt{R_{s}^{\prime 2}+1},
\end{aligned}
$$

and when needed, we have $V_{s}=U_{s}+2 R_{s}, u_{s}=v_{s}-2 x_{*}\left(R_{s}\right)$.

As in the null case, we will calculate the semiclassical flux in this spacetime, for three classes of observers $\mathcal{O}_{1}, \mathcal{O}_{2}$ and $\mathcal{O}_{3}$. Observers $\mathcal{O}_{1}$ and $\mathcal{O}_{2}$ always remain outside the shell, while observers $\mathcal{O}_{3}$ remain inside the shell until some time. More precisely, $\mathcal{O}_{1}$ correspond to $r=$ const observers with $r>R_{0}, \mathcal{O}_{2}$ are free-fall observers who cross the event horizon and fall into the singularity, and $\mathcal{O}_{3}$ are chosen to be $r$ =const observers inside the shell, who are tracked 


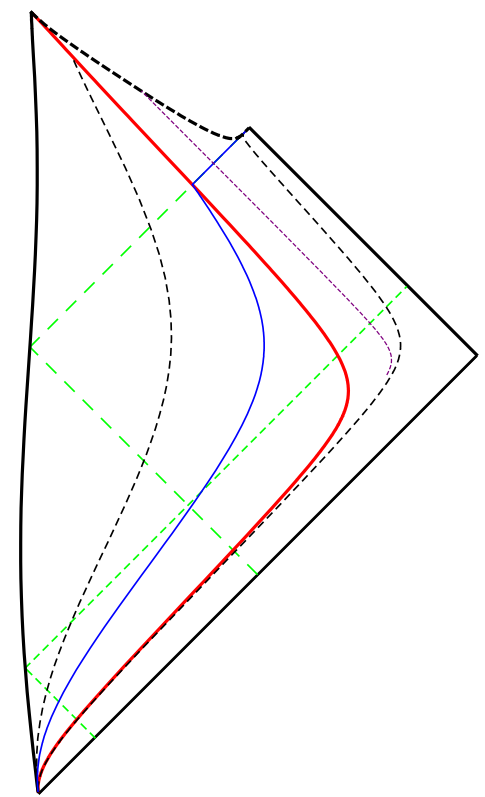

FIG. 3: Penrose diagram for timelike collapse of a shell, with $R_{0}=6 M, 2 M \kappa=0.3$. The origin (leftmost thick black curve) remains regular until the shell collapses to the singularity (thick dashed black). Various lines correspond to the shell trajectory (thick solid red), the surface $r=2 M$ (thin solid blue), the last escaper null ray (long dashed green), the ray which enters at $\tau=0$ (short dashed green), and example trajectories of observers $\mathcal{O}_{1}\left(r=6.15 M\right.$, exterior short dashed black), $\mathcal{O}_{2}(\beta=-0.5$, $\gamma=40.4 M$, fine dashed purple) and $\mathcal{O}_{3}(r=M$, interior short dashed black $)$.

until the time when the shell crosses their location. Figure 3 shows the Penrose diagram for this spacetime, with example trajectories of $\mathcal{O}_{1}, \mathcal{O}_{2}$ and $\mathcal{O}_{3}$ observers also shown. Recall from Section IB that the modes corresponding to the vacuum state $\mid$ in $\rangle$ for this spacetime, are completely fixed by requiring that (a) the modes on $\mathcal{I}^{-}$become $\sim e^{-i \omega v}$, and (b) the modes vanish at the origin $r=0$. Given these conditions, we can calculate the function $G$, which then determines the form of the conformal factor used to calculate the flux in Eqn. (6).

\section{Flux for observers $\mathcal{O}_{1}$}

The calculation in this subsection is well known in the literature (see, e.g. Ref. [3]). We will nevertheless display some relevant details which demonstrate the general procedure followed in the subsequent calculations. For observers $\mathcal{O}_{1}$ with constant $r$ who use the Eddington $u$ as their natural retarded time, the function $G(u)$ can be parametrized by $\tau$, with $u=u_{s}(\tau)$, and is given by

$$
G(\tau)=v_{s}\left(\tau_{1}(\tau)\right) ; V_{s}\left(\tau_{1}\right)=U_{s}(\tau),
$$

i.e., a ray which exits the shell at $\tau$, must have entered at a time $\tau_{1}$ determined by the second equation in (18), which is just the reflection condition. $G$ is then by definition the value of $v$ at the entry point $\tau_{1}$, and is treated as a function of $\tau$. Further note that

$$
\frac{d G}{d u}=\frac{d v_{s}\left(\tau_{1}\right)}{d \tau_{1}} \frac{d \tau_{1}}{d \tau} \frac{1}{\left(d u_{s} / d \tau\right)}=\tau_{1}^{\prime} v_{s}^{\prime}\left(\tau_{1}\right) / u_{s}^{\prime},
$$

where we have introduced the convenient shorthand notation of using a prime to denote a derivative with respect to the argument, and evaluation of all functions will be at their natural argument $\tau$, unless explicitly stated as in the case of $v_{s}^{\prime}\left(\tau_{1}\right)$. We will use this type of analysis for the observers $\mathcal{O}_{2}$ and $\mathcal{O}_{3}$ as well.

The (outgoing) flux $\mathcal{F}$ for these observers is independent of $r$, and is given by (see also Ref. [3])

$$
\mathcal{F}(u)=-\left\langle T_{t}^{r}\right\rangle=\left\langle T_{u u}\right\rangle-\left\langle T_{v v}\right\rangle=F_{u}\left[\frac{d G}{d u}\right],
$$




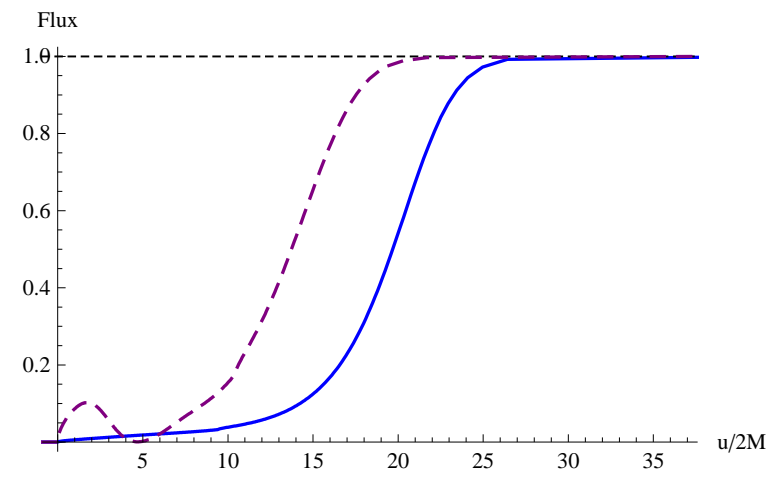

FIG. 4: The flux (normalized by the Hawking value $\left.(\pi / 12) T_{H}^{2}\right)$ as a function of $u$, for the timelike horizon-forming trajectory with $R_{0}=8 M$, for $r=$ const observers outside the shell. The two curves correspond to $2 M \kappa=0.1$ (solid blue) and $2 M \kappa=0.3$ (dashed purple). The initial behaviour of the flux depends on the trajectory details, but eventually the flux attains the constant Hawking value (short dashed black line).

where the derivative operator on the extreme right was defined in Eqn. (77). Using the relations in Eqns. (8), and parametrizing again using $\tau$, we can rewrite this as

$$
\mathcal{F}(\tau)=\frac{1}{u_{s}^{\prime 2}}\left\{-F_{\tau}\left[u_{s}^{\prime}(\tau)\right]+\left.\tau_{1}^{\prime 2}\left(F_{y}\left[v_{s}^{\prime}(y)\right]\right)\right|_{y=\tau_{1}(\tau)}+F_{\tau}\left[\tau_{1}^{\prime}(\tau)\right]\right\} .
$$

The expression on the right can be evaluated numerically, with the only nontrivial part being the inversion involved in determining $\tau_{1}(\tau)$. The result is shown in Fig. 4, where the flux is plotted as a function of $u$. As expected, this flux attains a constant value $(\pi / 12) T_{H}^{2}$ at late times.

\section{Flux for observers $\mathcal{O}_{2}$}

We take observers $\mathcal{O}_{2}$ to be freely falling into the horizon and eventually into the singularity. Defining the timelike and spacelike Kruskal coordinates

$$
t_{\mathrm{K}}=\frac{1}{2}(\mathcal{V}+\mathcal{U}) ; r_{\mathrm{K}}=\frac{1}{2}(\mathcal{V}-\mathcal{U}),
$$

such an observer can be described as moving at constant velocity in the $\left(t_{\mathrm{K}}, r_{\mathrm{K}}\right)$ coordinates, so that

$$
r_{\mathrm{K}}=\beta t_{\mathrm{K}}+\gamma / 2,
$$

for some constant $\gamma$ and $\beta<1$. This trajectory can also be parametrized using the shell's proper time $\tau$, and we ensure that this observer always remains outside the shell by choosing an appropriate $\gamma$ and tracking the trajectory only after a fixed time $\tau_{\mathrm{ob}, \mathrm{min}}$. (Recall that for the eternal Schwarzschild black hole, a trajectory such as Eqn. (23) corresponds to an observer who pops out of a white hole at $t=-\infty$, reaches a maximum radius $r=r_{\max }$, and falls into the horizon at $t=+\infty$.) It is convenient to write the trajectory in terms of the $r$ and $\mathcal{V}$ coordinates, as $r=R_{\mathrm{ob}}(\tau), \mathcal{V}=\mathcal{V}_{\mathrm{ob}}(\tau)$, where

$$
\begin{aligned}
\mathcal{V}_{\mathrm{ob}}(\tau) & =\left((1+\beta) \mathcal{U}_{s}+\gamma\right) /(1-\beta), \\
4 e^{R_{\mathrm{ob}}}\left(1-R_{\mathrm{ob}}\right) & =\mathcal{U}_{s} \mathcal{V}_{\mathrm{ob}},
\end{aligned}
$$

where we have used the relation between $r$ and the $\operatorname{Kruskal} \mathcal{U}, \mathcal{V}$ given by $\mathcal{U V}=4 e^{r}(1-r)$, and $\mathcal{U}_{s}(\tau)$ is the trajectory equation which can be obtained from the matching conditions on the shell,

$$
\mathcal{U}_{s}(\tau)=2 e^{R_{s}-v_{s} / 2}\left(1-R_{s}\right) .
$$

The outgoing flux $\mathcal{F}$ for such an observer becomes

$$
\mathcal{F}=\left.n_{a}\left\langle T_{b}^{a}\right\rangle u^{b}\right|_{\mathrm{ob}}=-\left.\left(r e^{r}\left\{\left\langle T_{\mathcal{V} \mathcal{V}}\right\rangle-\left\langle T_{\mathcal{U U}}\right\rangle\right\}\right)\right|_{\mathrm{ob}},
$$




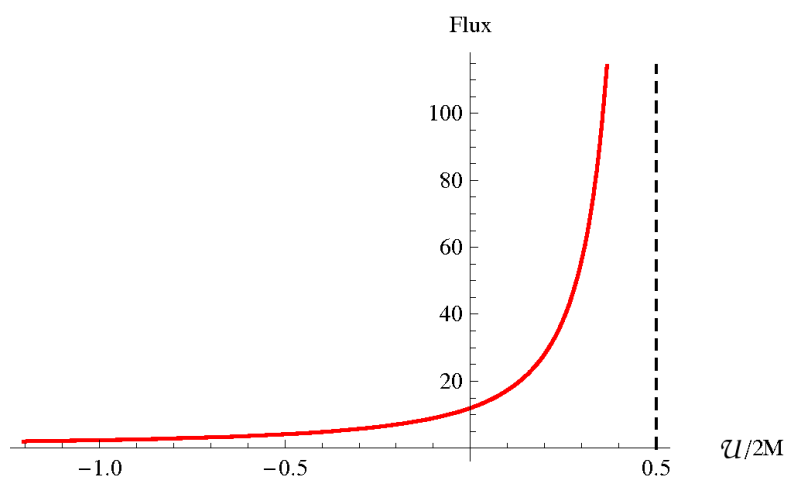

FIG. 5: The normalized flux (red solid line) for the timelike horizon-forming trajectory with $R_{0}=10 M$ and $2 M \kappa=0.1$, for a freely falling observer with $\beta=-0.75$ and $\gamma=27.7 M$. The flux is plotted as a function of retarded Kruskal time $\mathcal{U}$, with horizon formation occurring at $\mathcal{U}=0$. The vertical dashed line indicates the time at which this observer hits the singularity

so that the flux normalized by the Hawking value, denoted $\widetilde{\mathcal{F}}$, is

$$
\widetilde{\mathcal{F}}=-\left.4\left[\frac{e^{r}}{\mathcal{V}^{2} r^{2}}\left(\frac{3}{r}-4\right)-\frac{\mathcal{V}^{2}}{16 r} e^{-r}\left(1+\frac{2}{r}+\frac{3}{r^{2}}\right)\right]\right|_{\mathrm{ob}}+\left.192 \pi\left(r e^{r} F_{\mathcal{U}}\left[\frac{d G}{d \mathcal{U}}\right]\right)\right|_{\mathrm{ob}} .
$$

Note that while it seems analytically easier to parametrize this trajectory using the Kruskal coordinate $\mathcal{U}$, numerically it is more convenient to use the shell proper time, due to the appearance of the function $G$ in the flux. Since we are parametrizing the observer's trajectory using the shell's proper time, $r$ and $\mathcal{V}$ in Eqn. (27) will be replaced by $R_{\mathrm{ob}}(\tau)$ and $\mathcal{V}_{\mathrm{ob}}(\tau)$ respectively, and the last term involving the $F$ operator has a form identical to the RHS of Eqn. (21), with $u_{s}(\tau)$ replaced with $\mathcal{U}_{s}(\tau)$.

This flux is also straightforward to compute numerically, and the results are shown in Fig. [5, for a shell trajectory with $R_{0}=10 M$ and $2 M \kappa=0.1$, for an observer with $\beta=-0.75$ and $\gamma=27.7 M$. We see that while the flux is small (with magnitude comparable to the Hawking flux) as long as the observer remains outside the horizon, and remains finite at horizon crossing as well, once the observer approaches the singularity, the flux increases without bound. This is also obvious from the expression (27), where the first term in square brackets is singular as $r \rightarrow 0$.

\section{Flux for observers $\mathcal{O}_{3}$}

We now turn to the interior Minkowski region, and consider the flux measured by $r=$ const observers during the time that they remain inside the shell. We do not track such observers once the shell has crossed them, since this event is expected to be singular for such observers even classically, and we have not been accounting for the classical stress-tensor of the shell in our analysis. Unlike the null case, here we will see that interior observers do detect a nonzero flux, which is a consequence of the difference in global structure of $\mathcal{I}^{-}$in the two cases.

The $r=$ const observers inside the shell use $T=(V+U) / 2$ as their natural proper time. For any event $\mathcal{P}=\left(U_{*}, V_{*}\right)$ along the trajectory of such an observer, there are two events $\mathcal{P}_{1}$ and $\mathcal{P}_{2}$ relevant for the flux calculation. $\mathcal{P}_{1}$ is defined as the entry point (into the shell) of a ray which, after reflection at the origin, becomes the outgoing ray $U=U_{*}$. $\mathcal{P}_{2}$ is similarly the entry point of the ingoing ray which after entering the shell becomes $V=V_{*}$. Let $\tau_{1}$ and $\tau_{2}$ be the values of the shell proper time corresponding to the events $\mathcal{P}_{1}$ and $\mathcal{P}_{2}$ respectively. In terms of $(T, r)$ we have

$$
V_{s}\left(\tau_{1}\right)=U_{*}=T-r ; V_{s}\left(\tau_{2}\right)=V_{*}=T+r .
$$

The modes of the $\mid$ in $\rangle$ vacuum are then given by $\sim e^{-i \omega v_{s}\left(\tau_{2}\right)}-e^{-i \omega v_{s}\left(\tau_{1}\right)}$. Using the CFT rules for calculating the flux, it is then not hard to show that the normalized outgoing flux $\widetilde{\mathcal{F}}=\left(\left\langle T_{U U}\right\rangle-\left\langle T_{V V}\right\rangle\right) /\left(\pi T_{H}^{2} / 12\right)$ for such observers is given by

$$
\widetilde{\mathcal{F}}=\mathcal{S}\left(\tau_{1}\right)-\mathcal{S}\left(\tau_{2}\right)
$$

where the function $\mathcal{S}(\tau)$ is defined as

$$
\mathcal{S}(\tau)=\frac{192 \pi}{V_{s}^{\prime 2}(\tau)}\left(F_{\tau}\left[v_{s}^{\prime}(\tau)\right]-F_{\tau}\left[V_{s}^{\prime}(\tau)\right]\right)
$$




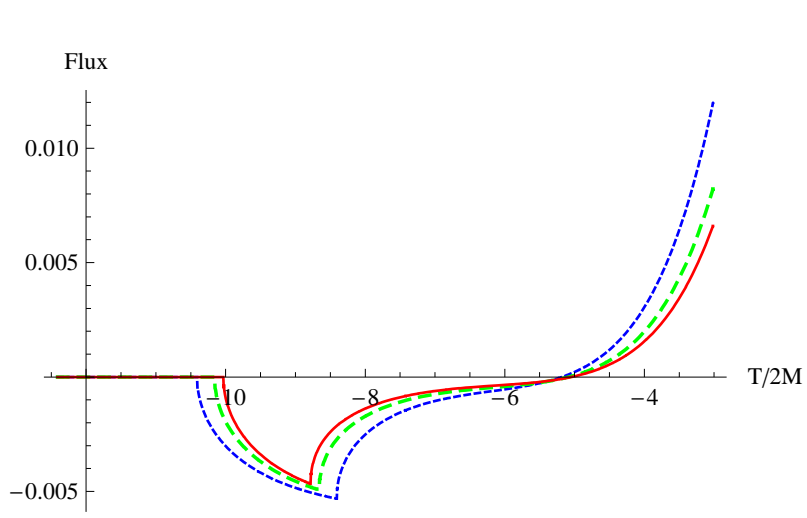

(a)

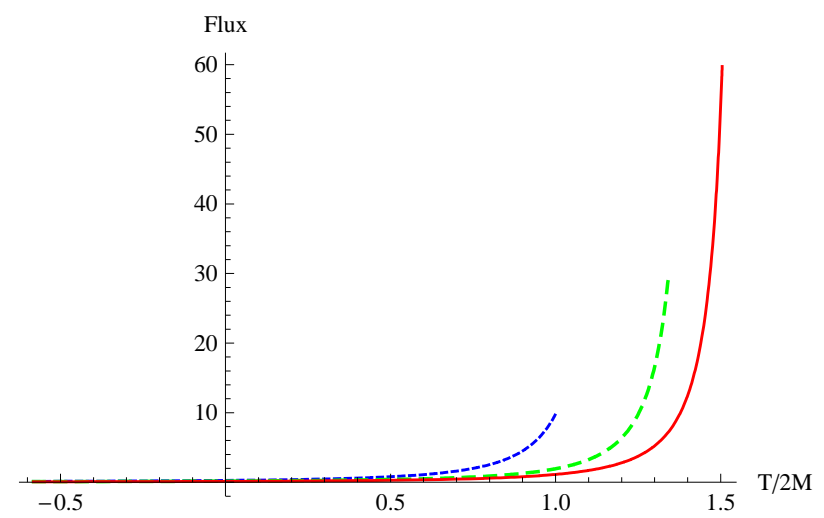

(b)

FIG. 6: The normalized flux for the timelike horizon-forming trajectory with $R_{0}=10 M$ and $2 M \kappa=0.1$, for three $r=$ const observers inside the shell, as a function of Minkowski time $T$. The curves correspond to : $r=1.25 M$ (solid red), $r=1.5 M$ (long dashed green), $r=2 M$ (short dashed blue). The left panel shows the initial, trajectory dependent phase, and the right panel shows the later phase. Each trajectory is tracked until the shell crosses it.

Using Eqn. (28) the flux can be expressed as a function of time $T$, for an observer with $r=$ const. The structure of the function $\mathcal{S}$ shows that the flux in the interior will remain zero as long as the shell trajectory satisfies $V_{s} \propto v_{s}$, which is the case until $\tau=0$. As a result, observers will see no flux as long as they are to the left of the ingoing ray which enters at $\tau=0$. (See the Penrose diagram Fig. 3. This is in fact true outside the shell as well.) This is also confirmed by Fig. 6 which shows the flux as a function of time for three $r=$ const observers. We see that after an initial complicated phase which depends on the trajectory details, the flux becomes positive and steadily increases in magnitude until the time the shell crosses the observer. This situation holds regardless of whether the shell crosses the observer before or after forming the event horizon, although the flux at shell crossing is larger for observers who are closer to the origin.

\section{B. Asymptotic trajectory}

In this section we discuss a class of trajectories first described in Ref. [13], which are timelike throughout and asymptotically approach a final radius larger than the Schwarzschild radius of the shell. Classically the interior geometry is Minkowski spacetime and the exterior is Schwarzschild spacetime, with no event horizon. Although such a trajectory was discussed in detail in Ref. [13], there are two reasons for returning to it here. Firstly, as mentioned earlier, the treatment in Ref. [13] was mainly analytical and hence the inversions required to calculate $G(u)$ could not be performed. This can now be done in our current numerical setup, and we will display results that corroborate those in Ref. 13]. Secondly, a numerical analysis allows us to further explore the semiclassical environment of this trajectory. Specifically, we will show below that such trajectories will generically give rise to a pulse of radiation which travels inwards, reflects at the origin and eventually reaches an external observer at late times. Mathematically, this new feature arises solely from the functional inversions at the shell boundary, and was (not surprisingly) missed in Ref. [13].

The asymptotic trajectory is parametrized by Eddington retarded time $u$ (which is a good parameter everywhere since there is no horizon), and is described by the following prescription, borrowed from Ref. [13] :

$$
U_{s}^{\prime}(u)=\epsilon+\left(\left(1-\frac{1}{R_{0}}\right)^{1 / 2}-\epsilon\right) e^{-\alpha(u)} \equiv \epsilon+A e^{-\alpha(u)},
$$

where, as before, the prime is a derivative with respect to the argument and we are working with the nondimensionalised form of the coordinates. Here $0<\epsilon<\left(1-1 / R_{0}\right)^{1 / 2}$ is a fixed constant,

$$
\alpha(u)=\frac{\theta(u)}{2} \int_{0}^{u} d \tilde{u} h(\tilde{u})
$$




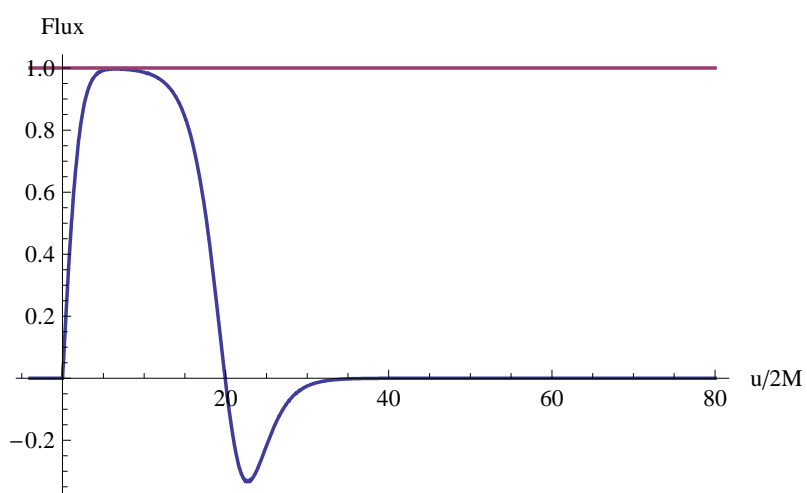

(a)

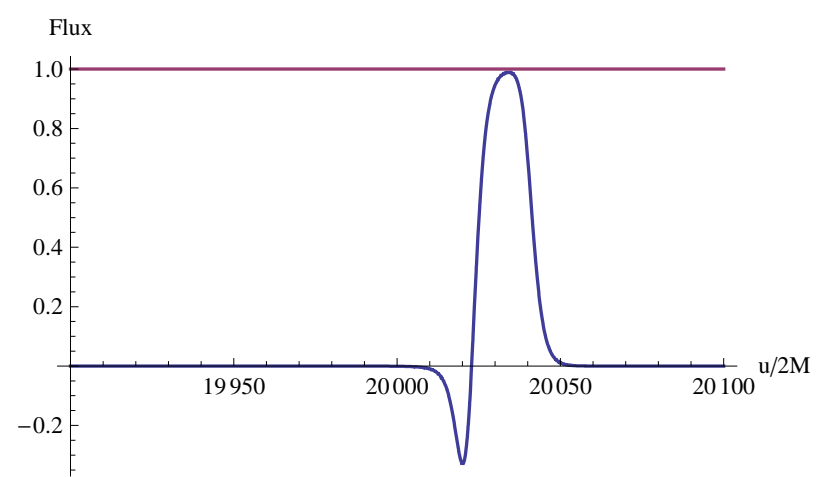

(b)

FIG. 7: The normalized exterior flux as a function of $u$, for the timelike asymptotic trajectory with $R_{0}=3 M, \epsilon=10^{-4}$ and $2 M \lambda=0.25$. The left panel shows the initial phase which shows a thermal behaviour followed by an exponential decay. The right panel shows the flux for the same configuration at a much later time. The second, "pseudo-thermal" phase can be traced back to a pulse generated at an inflection point of the trajectory, as discussed in the text.

where $\theta(u)$ is the Heaviside step function and $h(u)$ is chosen to have the following asymptotic behaviour

$$
\begin{gathered}
h(u)=0, u \leq 0 \quad ; \quad h^{\prime}(u)=0, u \leq 0, \\
h(u \rightarrow \infty) \rightarrow 1 ; \quad h^{\prime}(u \rightarrow \infty) \rightarrow 0,
\end{gathered}
$$

and we require the asymptotic values for $h$ and $h^{\prime}$ to be achieved exponentially fast, with a time scale determined by $M$. For this paper, we choose $h(u)=\theta(u) \tanh ^{2}(\lambda u)$ for some constant $\lambda$. This is slightly different from the choice in Ref. [13]; this change does not alter the qualitative behaviour of the trajectory and was made to improve the numerical behaviour of various subsequent functions. The trajectory radius $R_{s}$ satisfies the equation

$$
2 R_{s}^{\prime}\left(1-U_{s}^{\prime}\right)=U_{s}^{\prime 2}-\left(1-\frac{1}{R_{s}}\right)
$$

so that $R_{s}$ starts at a constant radius $R_{0}$, falls inwards and asymptotically approaches a final radius $R_{f} \equiv 1 /\left(1-\epsilon^{2}\right)$. The Penrose diagram for such a trajectory was shown in Ref. [13] (see their Fig. 3).

We again consider two classes of observers : exterior observers at some constant radius $r>R_{0}$, and interior observers at some constant radius $r<R_{f}$. For brevity we ignore observers who are initially inside the shell and eventually come into the Schwarzschild region, since such observers will not see any effects that are absent for the previous two classes. For the exterior observers, the normalized flux is easily seen to be identical in expression to the one calculated for the previous trajectory

$$
\widetilde{\mathcal{F}}=(192 \pi) F_{u}\left[\frac{d G}{d u}\right]
$$

where

$$
G(u)=v_{s}\left(u_{1}(u)\right) ; V_{s}\left(u_{1}\right)=U_{s}(u),
$$

which is familiar from earlier, with $\tau$ being replaced by $u$. The expression for $F_{u}[d G / d u]$ is somewhat simpler in terms of $u$,

$$
F_{u}\left[\frac{d G}{d u}\right]=\left.u_{1}^{\prime 2}(u)\left(F_{y}\left[v_{s}^{\prime}(y)\right]\right)\right|_{y=u_{1}(u)}+F_{u}\left[u_{1}^{\prime}(u)\right] .
$$

Although these expressions may appear different from Eqn. (27) of Ref. [13], the two sets are in fact equivalent. The specific splitting used in Ref. [13] was conceptually easier to tackle, while the expressions above are easier to handle numerically.

Since the dependence of the flux on the model parameters was discussed in detail in Ref. [13], here we will simply display results for a choice of parameter values that enhances the effects we want to focus on. The effects themselves 


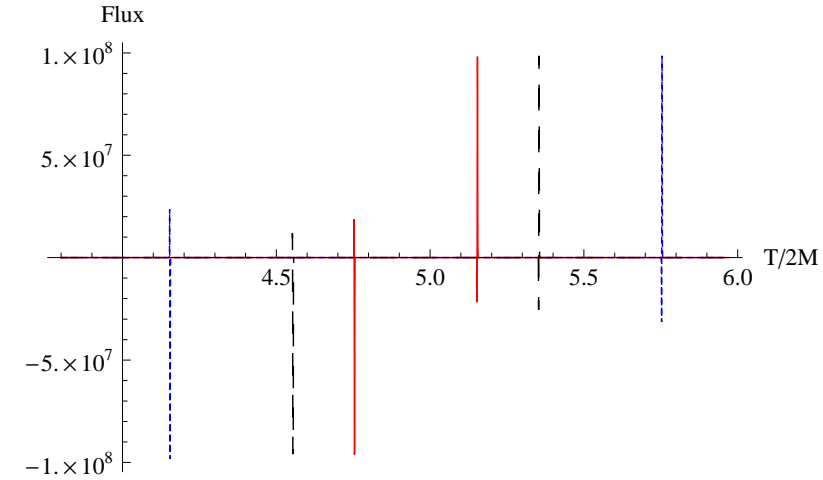

(a)

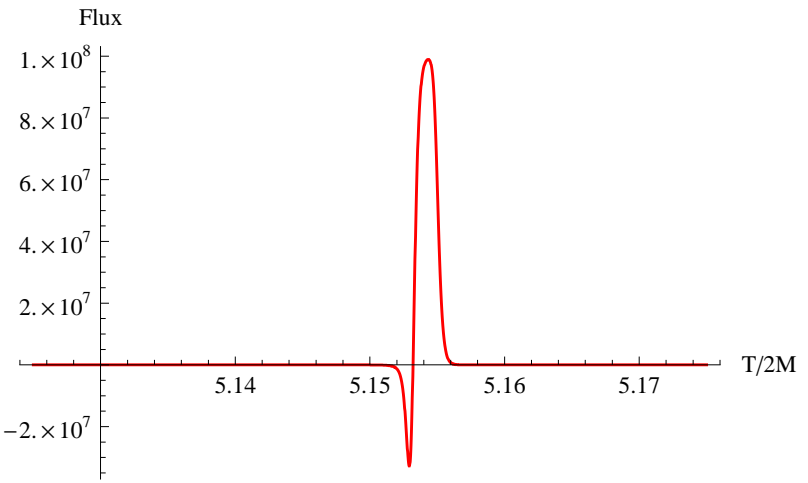

(b)

FIG. 8: The normalized interior flux as a function of Minkowski time $T$, for the timelike asymptotic trajectory with $R_{0}=3 M$, $\epsilon=10^{-4}$ and $2 M \lambda=0.25$. The left panel shows the flux seen by three observers at constant $r: r=0.4 M$ (solid red), $r=0.8 M$ (long dashed black) and $r=1.6 M$ (short dashed blue). The right panel shows details of the pulse (shown on the outgoing leg for the observer with $r=0.4 M)$.

are generic and do not depend on the specific parameter choices we make. Figure 7 shows the exterior flux as a function of $u$, for a trajectory with $R_{0}=3 M, \epsilon=10^{-4}$ and $2 M \lambda=0.25$. As expected for such a trajectory, we see an initial phase in the flux which closely resembles that in the conventional trajectory : the asymptotic trajectory initially behaves as if it were heading towards horizon formation. Eventually however, the effects of the nonzero $\epsilon$ are felt and the flux exponentially decays to zero. This part is captured in the left panel of the figure, and was also described in Ref. [13]. The right panel shows the flux for the same trajectory, but at much later times. We see a second pseudo-thermal phase (more on this nomenclature later), which rises from zero, reaches and stays at the Hawking value, and then also exponentially decays. The origin of this second phase will become clear soon, when we discuss the flux seen by the interior observers.

For the interior observers, the flux calculation again proceeds identically to that for the horizon-forming trajectory. In fact, it is easily verified that the function $\mathcal{S}$ defined in Eqn. (30) is parametrization independent, so that the normalized interior (outgoing) flux for the present case is given by

$$
\begin{aligned}
\widetilde{\mathcal{F}} & =\mathcal{S}\left(u_{1}\right)-\mathcal{S}\left(u_{2}\right), \\
\mathcal{S}(u) & =\frac{192 \pi}{V_{s}^{\prime 2}(u)}\left(F_{u}\left[v_{s}^{\prime}(u)\right]-F_{u}\left[V_{s}^{\prime}(u)\right]\right),
\end{aligned}
$$

with $u_{1}$ and $u_{2}$ defined in terms of $(T, r)$ via

$$
V_{s}\left(u_{1}\right)=T-r ; V_{s}\left(u_{2}\right)=T+r .
$$

As before, the flux will be nonzero only on the right of the ingoing ray that enters at $u=0$, since in the region to the left of this ray we have $V_{s} \propto v_{s}$. Figure 8 shows the flux seen by three observers at three different constant radii $r$, for the same configuration as in Fig. 17. Each observer sees a strong incoming pulse of radiation and an outgoing pulse at a later time. By studying the time intervals between the pulses seen by various observers, it is possible to show that there is only one pulse which starts at the shell at a certain time, travels inwards and gets reflected at the origin, and is then seen by the observers again on its way out. This is also consistent with the fact that the second pulse seen by any interior observer is the precise mirror image of the first (although this is not obvious from the figure). The right panel in Fig. 8 shows the detailed structure of the pulse.

This discussion also suggests that this pulse should be detected by exterior observers as well, and in fact one finds that the second pseudo-thermal phase referred to earlier, is precisely the remnant of this pulse after it exits the shell. Further, the origin of this pulse can be traced to the time interval in which the behaviour of $U_{s}$ changes from $U_{s} \sim\left(1-1 / R_{0}\right)^{1 / 2} u$ to $U_{s} \sim \epsilon u$ (see Eqn. (31)). To visually demonstrate this we define the time $u_{\text {change }}$ such that the slope $U_{s}^{\prime}\left(u_{\text {change }}\right)=e \epsilon$, i.e. one $e$-folding away from its final asymptotic value. In Fig. 9, the vertical dashed line

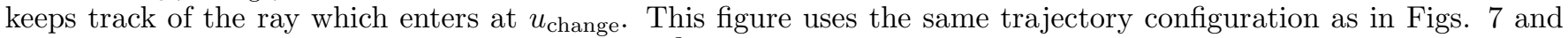
8 except for the value of $\epsilon$, which is now $\epsilon=10^{-3}$. Figure 9 when compared with Figs. 7 and 8 also suggests that the pulse height in the interior scales approximately as $\epsilon^{-2}$, which also follows from considering the structure of $\mathcal{S}$, and that the time at which the second phase occurs in the exterior, scales roughly as $\epsilon^{-1}$. This is also supported by a more detailed numerical analysis (not shown). 


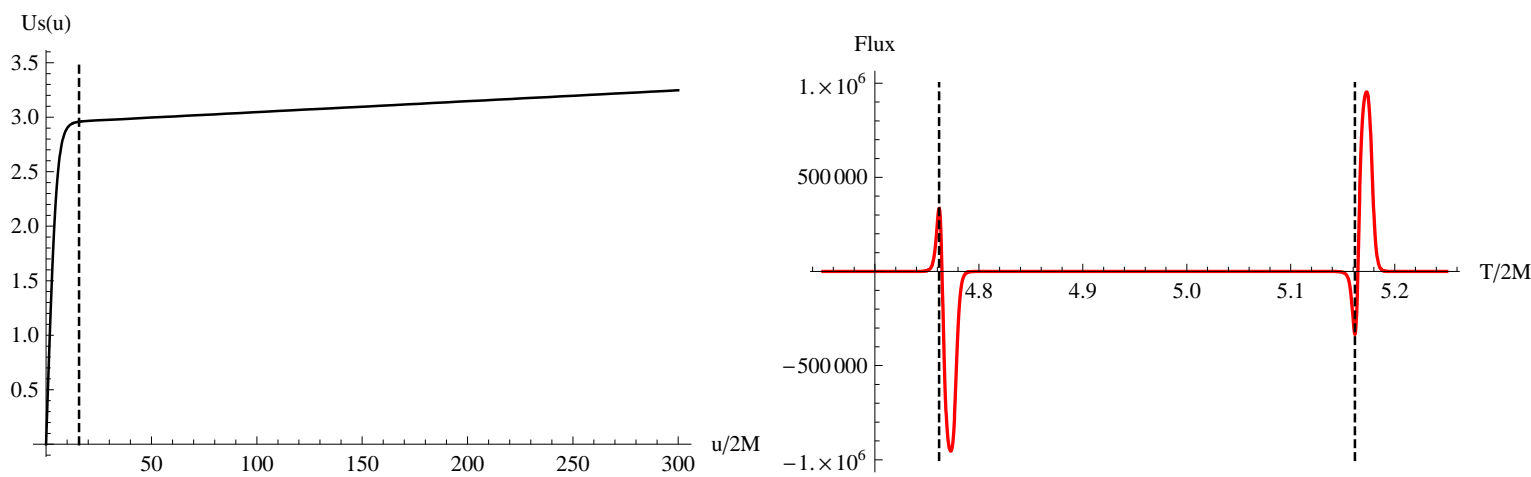

(a)

(b)

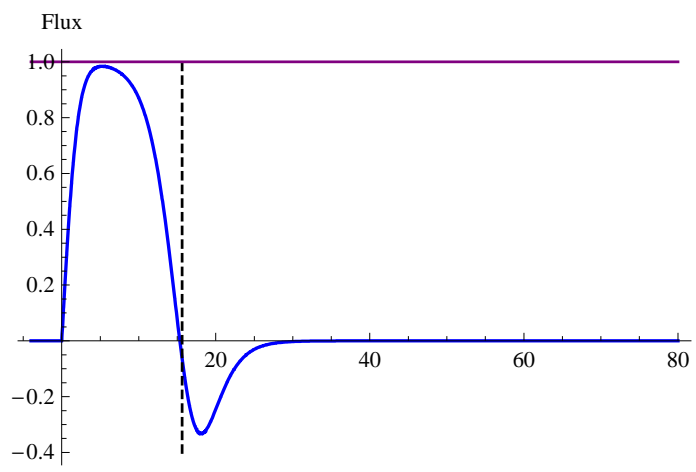

(c)

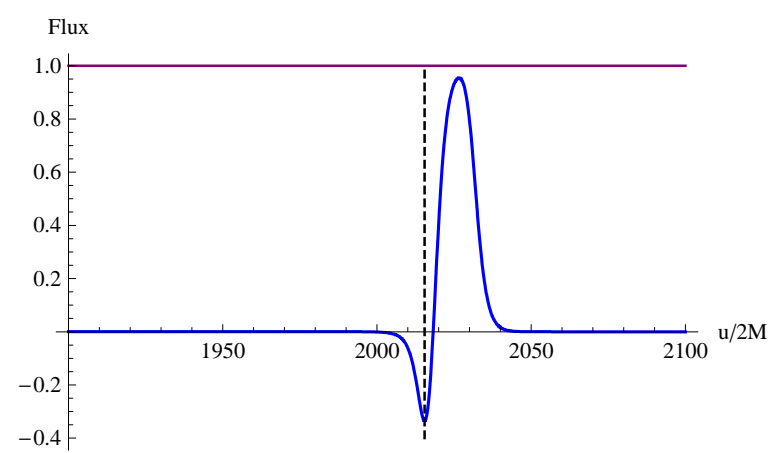

(d)

FIG. 9: The behaviour of the pulse which arises in the interval when $U_{s}$ changes its behaviour (panel (a)), for the asymptotic trajectory configuration $R_{0}=3 M, \epsilon=10^{-3}$ and $2 M \lambda=0.25$. The vertical dashed line in panel (a) marks $u=u_{\text {change }}$, and the

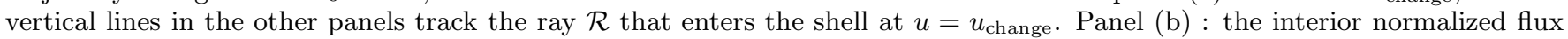
for the observer at $r=0.4 M$. Vertical lines mark the times when this observer meets the ray $\mathcal{R}$, before and after reflection. Panel (c) : The initial phase of the exterior flux. Vertical line marks the ray $\mathcal{R}$ on the way in. Panel (d) : The late phase of the exterior flux. Vertical line marks the ray $\mathcal{R}$ on the way out.

One significant aspect of the pulse of radiation is that it is very sharply peaked in the interior of the shell, and becomes diffuse once it exits to the exterior. In fact one can check that the height of the pulse in the exterior never exceeds the Hawking value, instead the width increases with decreasing $\epsilon$, and the flux in the exterior pulse spends a longer time at the Hawking value. This is why we referred to this phase as "pseudo-thermal" earlier. It will be very interesting to see the signatures left by this pulse in the spectrum of particles in various regions. This however requires a calculation of the Bogolubov coefficients, which is complicated by the presence of numerical inversions, and is beyond the scope of this work. Another tantalizing prospect is that such a pulse could possibly lead to astrophysical signatures in objects which undergo phases of halted collapse without forming a horizon. This would, however, require some kind of amplification mechanism, since the total amount of gravitational energy released in the pulse appears to be very small (while the pulse height can become large, the pulse width decreases with decreasing $\epsilon$ ).

\section{DISCUSSION}

We have explored in detail the semiclassical environment of a collapsing shell of matter. Our main objective was to study the semiclassical flux observed by a variety of observers, both inside and outside the shell. We have studied two types of trajectories : those which form a horizon and those which do not.

For the horizon forming trajectories, we reproduced the well known result of an asymptotically constant flux seen by observers outside the horizon. Additionally we demonstrated that freely falling observers measure an outgoing flux which generically increases without bound as they approach the singularity. Our calculation assumed zero backreaction, and it is possible that the infinity we see in our flux can somehow be cured if backreaction were correctly accounted for throughout. It is hard to guess how exactly this might happen, since a self-consistent calculation of a black hole geometry in the presence of backreaction at late stages of evaporation, does not exist. It is possible that 
the resolution of this problem is linked to the more general problem of the singularity itself.

We also saw, at least in the timelike case, that inertial observers inside the shell also see a nonzero outgoing flux, which steadily increases until the time they exit the shell. In the context of backreaction, this has consequences for all arguments built on the assumption that the interior geometry of the shell remains that of Minkowski spacetime throughout. This assumption was made for example in Ref. [13] while arguing that the semiclassical backreaction can at best delay the formation of the event horizon by a short amount of time. While it is unlikely that including a flux of the same order of magnitude as the Hawking flux would overturn the conclusions of the arguments in Ref. [13], it is nevertheless important to rigorously account for this additional flux and build a complete picture. Specifically, it is interesting to ask what the self-consistent geometry in the interior will be, after accounting for backreaction. We will return to this question in future work.

In the case of the asymptotic trajectory which halts collapse before horizon formation, we corroborated the analysis of Ref. [13] by a full numerical calculation, and extended the results to include the flux seen by interior observers. In the process we uncovered an interesting, generic feature of such trajectories, in the form of a pulse of radiation which is sharply peaked in the interior but appears to thermalize as it emerges from the shell. It remains to be seen whether this feature might lead to astrophysically interesting signatures.

For both types of trajectories discussed here, it will be interesting to determine the nature of the particle spectrum, for which we need to calculate the Bogolubov coefficients in the respective geometries. This is work in progress. We expect our current results to be useful in addressing the broader issues mentioned in the Introduction, specifically questions related to the interpretation of thermodynamic variables such as entropy, for a variety of classes of observers, and in studying the role and nature of the semiclassical backreaction.

\section{Acknowledgments}

We wish to thank T. Padmanabhan for suggesting this project, and for many insightful discussions. AP thanks Dawood Kothawala for useful discussions, and gratefully acknowledges hospitality at IUCAA where most of this work was completed.

[1] S Hawking, Nature 248, 30 (1974); Commun. Math. Phys. 43, 199 (1975).

[2] W G Unruh, Phys. Rev. D14, 870 (1976).

[3] N D Birrell and P C W Davies, Quantum Fields in Curved Space, Cambridge Univ. Press, Cambridge (1984).

[4] P C W Davies, S A Fulling and W G Unruh, Phys. Rev. D13, 2720 (1976).

[5] D N Page, Phys. Rev. D13, 198 (1976);

N G Sanchez, Phys. Rev. D18, 1030 (1978);

K W Howard, Phys. Rev. D30, 2532 (1984);

J M Bardeen, Phys. Rev. Lett. 46, 382 (1981);

J W York, Jr., Phys. Rev. D28, 2929 (1983);

For a pedagogical review and further references, see

M Visser, Int. J. Mod. Phys. D12, 649 (2003) arXiv:hep-th/0106111.

[6] For a review see

T Padmanabhan, Phys. Rept. 406, 49 (2005) arXiv:gr-qc/0311036.

[7] S Mathur, arXiv:0909.1038 (2009).

[8] G 't Hooft, arXiv:0909.3426 (2009);

C Kiefer, Quantum Gravity, Oxford Univ. Press, Oxford, UK (2007).

[9] R Brout, S Massar, R Parentani and Ph Spindel, Phys. Rept. 260, 329 (1995) arXiv:0710.4345.

[10] S Massar, Phys. Rev. D52, 5857 (1995) arXiv:gr-qc/9411039;

R Brout, et al., Phys. Rev. D52, 1119 (1995) arXiv:hep-th/9311019;

T Clifton, Class. Quant. Grav. 25, 175022 (2008) arXiv:0804.2635];

B A Brown and J Lindesay, Class. Quant. Grav. 25, 105026 (2008) arXiv:0710.2032;

A B Nielsen and D-h Yeom, arXiv:0804.4435 (2008);

G L Alberghi, R Casadio, G P Vacca and G Venturi, Phys. Rev. D64, 104012 (2001) arXiv:gr-qc/0102014.

[11] T Vachaspati, D Stojkovic and L M Krauss, Phys. Rev. D76, 024005 (2007) arXiv:gr-qc/0609024;

T Vachaspati and D Stojkovic, Phys. Lett. B663, 107 (2008) arXiv:gr-qc/0701096;

T Vachaspati, arXiv:0711.0006 (2007).

[12] C Barcelo, S Liberati, S Sonego and M Visser, Phys. Rev. D77, 044032 (2008) arXiv:0712.1130.

[13] A Paranjape and T Padmanabhan, Phys. Rev. D80, 044011 (2009) arXiv:0906.1768.

[14] R J Adler, J D Bjorken, P Chen and J S Liu, Am. J. Phys. 73, 1148 (2005) arXiv:gr-qc/0502040.

[15] A Fabbri and J Navarro-Salas, Modeling black hole evaporation, Imperial Coll. Press, London, UK (2005). 